\title{
Salvaging a tooth by hemisection
}

\author{
M Vikram ${ }^{1}$, AR Vivekananda Pai ${ }^{2}$ \\ Department of Conservative Dentistry and Endodontics \\ ${ }^{1}$ B.P.Koirala Institute of Health Sciences, Dharan, Nepal \\ ${ }^{2}$ Manipal College of Dental Sciences, Mangalore, India
}

\begin{abstract}
Introduction : Hemisection may be a valuable form of treatment for molar teeth when caries has progressed to the furcation, or where there is an extensive carious lesion extending subgingivally in one area of the root, making it impossible to place an adequate restoration in that area and the root is considered unrestorable. Case Presentation : When one of the roots of a multirooted teeth is beyond the scope of restoration, it becomes imperative to remove the diseased root. This case report is about the use of hemisection procedure which was carried out to salvage the mesial portion of the mandibular right first molar. Conclusion : The use of the procedure of hemisection can successfully eliminate the need for extraction of the whole tooth if only a part of it is unrestorable.
\end{abstract}

Keywords : hemisection, root caries, furcation

\section{Introduction}

A molar tooth with extensive root caries may be unsuitable for restoration. In such cases, the treatment options are limited and may include a partial denture or a dental implant to replace the missing tooth. Alternatively, if the decay is limited to one root, a hemisection procedure may be possible. This procedure represents a form of conservative dentistry, aiming to retain as much of the original tooth structure as possible. The results are predictable, and success rates are high if certain basic considerations are taken into account. ${ }^{1}$ Hemisection as the name implies is the division of a tooth and removal of the unwanted portion thereafter. ${ }^{2}$

The goal of this approach is either to remove single roots with a poor prognosis, for example, because of advanced attachment loss, endodontic treatment

Address for correspondence

Dr. Mannu Vikram, MDS

Assistant Professor

Department of Conservative Dentistry and Endodontics

B.P.Koirala Institute of Health Sciences, Dharan, Nepal

Email:mannu_vikram07@yahoo.com complications, root fracture, or subgingival root caries, or to remove plaque niches by transforming multirooted teeth with severe furcation involvement into single rooted teeth, thus improving access to oral hygiene measures. ${ }^{3}$ Also, hemisection procedure may be used to separate a geminated or a fused teeth. ${ }^{4,5,6}$ Hemisection of a maxillary or mandibular molar is often a means of retaining teeth needed for restorative abutments or occlusal support. This treatment can yield predictable results using proper diagnostic, endodontic, surgical, and prosthetic techniques. $^{7}$

\section{Case Report}

A 42-year-old man reported to the dental clinic with intermittent pain on the lower right side of the mouth. Also, the patient complained of extensive food impaction in the same region. Upon clinical examination, disto-proximal caries in relation to the mandibular right first molar was present which was extending subgingivally. The tooth was tender on percussion. A large amalgam restoration was present in relation to mandibular right second molar. The 
patient gave a history of root canal treatment with respect to the same tooth one year back. Upon radiographic examination, there was a coronal radiolucency extending upto the middle third of the distal root in relation to mandibular right first molar. There was radioopacity in the root canal and pulp chamber in relation to mandibular right second molar indicative of previous root canal treatment. Also, the radioopacity did not extend upto the full length of the root canal suggestive of deficient obturation (Fig. 1). Since, there was extensive root caries with respect to the distal root of 46, root canal treatment would not be feasible. So, a decision was taken to salvage only the mesial portion of the tooth and extract the distal portion by doing a hemisection. Thereafter, the edentulous space would be replaced by a fixed partial denture by incorporating 47 and the mesial portion of 46 as the abutment teeth. Therefore, a decision to do root canal treatment only for the mesial root of 46 was taken. Also, a decision to do a retreatment for 47 was taken.

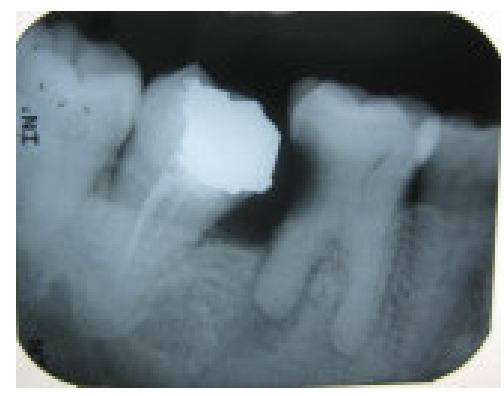

Fig. 1

Access opening was done with respect to 46 under rubber dam isolation. Working length was established and cleaning and shaping was done with a crown-down approach. The patient was recalled again after a week and obturation for the mesial root of 46 was done (Fig. 2). The core build up was done with Glass Ionomer Cement. Similarly, retreatment of 47 was done.

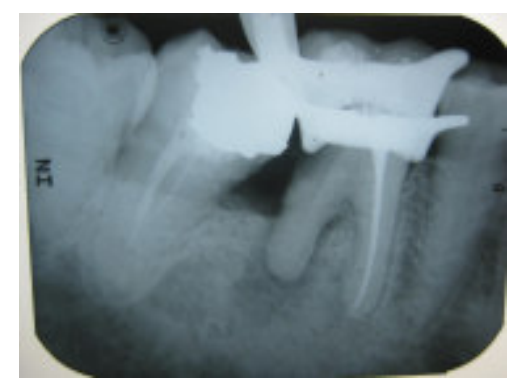

Fig. 2
Hemisection was done with respect to 46 using a long tapered fissure bur. The bur was used in a sawing motion in a bucco-lingual direction. During the procedure, radiographs were taken to verify the length of the cut. This was done to avoid injuring the furcal bone of the alveolar socket. The distal root of 46 was extracted by first using an elevator and then by using an extraction forceps. A post operative radiograph was then taken to verify that the entire root had been taken out and also to make sure that the extraction socket was clean of any foreign particles (Fig. 3). Further, after the extraction socket wound heals, a fixed partial denture is planned taking 47 as the distal abutment and the mesial portion of 46 as the mesial abutment.

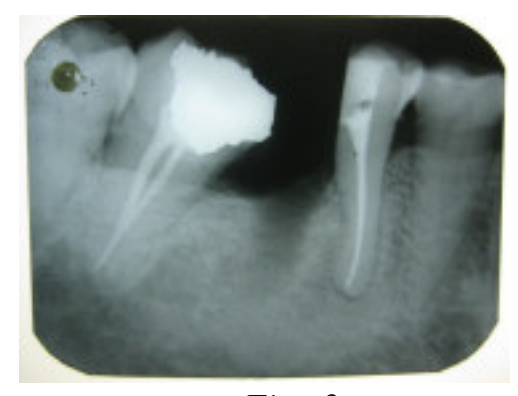

Fig. 3

\section{Discussion}

Restoring the extensively damaged dentition has always been a difficult decision-making process for the dental practitioner. Decisions to restore these teeth were primarily based on the fact that "fixed" teeth are typically better functionally than "removable" teeth and better esthetically than "no" teeth. ${ }^{8}$ Hemisection as the name implies is the division of a tooth and removal of the unwanted portion thereafter. This technique was performed on a mandibular first permanent molar. ${ }^{2}$

Hemisection may be a suitable alternative to extraction and implant therapy and should be discussed with patients during consideration of treatment options. ${ }^{9}$ Hemisection is a surgical method for treating lesions of two- and multi-rooted teeth. This procedure includes amputation of one or two lesioned roots together with the attached dental crown, leaving an endodontically treated root and the remaining dental crown prepared for future prosthetic restoration. ${ }^{10}$ Hemisection of mandibular molars may be a viable treatment option when vertical root fracture has occurred and the other root is healthy. ${ }^{11}$ 
Surgical hemisection of a tooth involves the deliberate excision of one or more roots and their associated coronal structure. This procedure generally is performed as an alternative to complete extraction on molars when their prognosis can be improved by removing roots that are significantly compromised. Traumatic hemisection is nondeliberate and describes complete fracture through a furcation, resulting in severance of a fragment from the tooth. ${ }^{12}$

For this patient, hemisection was selected for treatment of root decay in the terminal abutment of a fixed prosthesis. Implant therapy was considered but was not feasible; instead, a 3-unit fixed partial denture, extending from the hemisected molar to the second molar, was planned. The distal root was resected because of the location of the decay. The literature on distal root resection is limited; more often, this root is retained and the mesial root removed. However, the distal root is broader and straighter, making it more suitable as an abutment. The mesial root contains a longitudinal groove, which decreases its surface area and contraindicates the use of posts. ${ }^{9}$ Implant therapy is a predictable option with good functionality; however, in this case, the patient chose an alternative treatment because of financial considerations.

\section{Conclusion}

Hemisection may be a suitable alternative to extraction and implant therapy and should be discussed with patients during consideration of treatment options. Following careful clinical and radiographic examination, hemisection can be selected as an appropriate treatment alternative in cases where when one of the roots has a decay which is beyond the scope of restoration.

\section{References}

1. Sun-Young Kim, Sung Chul Choi,b and Younjoo Chung. Management of the fused permanent upper lateral incisor: a case report. Oral Surg Oral Med Oral Pathol Oral Radiol Endod 2011;111:649-652

2. Prabhu NT, Munshi AK. Hemisection of a permanent mandibular first molar: a treatment option for a vertically impacted second premolar. J Clin Pediatr Dent. 1996 Spring;20(3):233-5.
3. Gregory-george Zafiropoulos, Oliver Hoffmann, Adrian Kasaj, Brita Willershausen, Giorgio Deli, Dimitris N. Tatakis. Mandibular molar root resection versus implant therapy: a retrospective nonrandomized study.Journal of Oral Implantology vol. xxxv/no. two/2009

4. A. Braun,T. Appel, M. Frentzen. Endodontic and surgical treatmentof a geminated maxillary incisor. International Endodontic Journal, 36, 380386, 2003

5. Tugba Cetinbas, Suleyman Halil, Mehmet Okan Akcam, Saziye Sari, and Serap Cetiner. Hemisection of a fused tooth. Oral Surg Oral Med Oral Pathol Oral Radiol Endod 2007;104:e120-e124

6. Karacay, Seniz; Guven, Gunseli, Koymen, Ramazan. Management of a Fused Central Incisor in Association with a Macrodont Lateral Incisor: A Case Report Pediatric Dentistry, Volume 28, Number 4, July/August 2006 , pp. 336-340

7. Silverstein LH, Moskowitz ME, Kurtzman D, Shatz PC, Gornstein RA. Prosthetic considerations with periodontal root resective therapy, Part 2. Hemisections. Dent Today. 1999 Sep;18(9):86-9.

8. Cho GC. Evidence-based approach for treatment planning options for the extensively damaged dentition. J Calif Dent Assoc. 2004 Dec;32(12):983-90.

9. M. Najeeb Saad, Jorge Moreno, Cameron Crawford. Hemisection as an Alternative Treatment for Decayed Multirooted Terminal Abutment: A Case Report. JCDA June 2009, Vol. 75 , No. 5

10. Góra M. Usefulness of hydroxyapatite implants for the management of alveolar bone lesions at tooth hemisection sites]. Ann Acad Med Stetin. 2005;51(1):57-63.

11. Kurtzman GM, Silverstein LH, Shatz PC. Hemisection as an alternative treatment for vertically fractured mandibular molars. Compend Contin Educ Dent. 2006 Feb;27(2):126-9.

12. Rapoport RH, Deep P. Traumatic hemisection and restoration of a maxillary first premolar: a case report Gen Dent. 2003 Jul-Aug;51(4):340-2. 